\title{
Test of two Nb superstructure prototypes
}

J. Sekutowicz, P. Castro, A. Gössel, G. Kreps, R. Lange, A. Matheisen, W.-D. Möller, H.-B. Peters, D. Proch, H. Schlarb, S. Schreiber, S. Simrock, and M. Wendt

DESY, Notkestrasse 85, 22603 Hamburg, Germany

N. Baboi

SLAC, 2575 Sand Hill Road, Menlo Park, California 94025, USA

M. Ferrario

INFN, Via E. Fermi 40, 00044 Frascati, Italy

M. Huening

FNAL, MS 306, Batavia, Illinois 60510, USA

M. Liepe

Cornell University, Ithaca, New York 14853, USA

C. Pagani

INFN-Milano, Via Fratelli Cervi 201, 20090 Segrate, Italy

S. Zheng

Tsinghua University, 100084 Beijing, China

(Received 21 October 2003; published 20 January 2004)

An alternative layout of the TESLA linear collider (Technical Design Report, DESY Report No. 2001-011, 2001), based on weakly coupled multicell superconducting structures (superstructures), significantly reduces the investment cost due to a simplification in the rf system of the main accelerator. In January 1999, preparation of the beam test of the superstructure began in order to prove the feasibility of this layout. Progress in the preparation was reported frequently in Proceedings of TESLA Collaboration Meetings. Last year, two superstructures were installed in the Tesla Test Facility linac at DESY to experimentally verify methods to balance the accelerating gradient in a weakly coupled system, the stability of the energy gain for the entire train of bunches in macropulses, and the damping of higher order modes. We present results of the first cold and beam test of these two $\mathrm{Nb}$ prototypes.

DOI: 10.1103/PhysRevSTAB.7.012002

PACS numbers: 29.17.+w

\section{INTRODUCTION}

Superstructures (SSTs), chains of superconducting multicell cavities (subunits) connected by $\lambda / 2$ long tube(s), have been proposed as an alternative layout for the TESLA main accelerator [1,2]. There are two main advantages of the layout in comparison to the standard one, based on 9-cell cavities. The first, an economic advantage, is that structures made of more cells will reduce the number of fundamental power couplers (FPC) in the linac. Consequently, the number of all auxiliaries needed to distribute the rf power, like waveguides, bends, circulators, 3-stub transformers, loads, etc. can be reduced too. In addition, the layout reduces the amount of electronics controlling phase and amplitude of cavities in the linac and simplifies the design of cryomodules due to fewer openings for the FPCs. The second advantage is the increased filling of the linac tunnel with accelerating structures, since the distance between subunits is only $\lambda / 2$. The space saving can be significant. In the case of versions of SSTs discussed later, it is $\sim 1.8 \mathrm{~km}$. When the end energy of the collider is fixed one has a choice to keep the nominal gradient and to make the accelerator shorter or to keep its length and to lower the nominal gradient. The best way to use this additional space is a trade-off between the investment cost and the performance of the collider. In superstructures, unlike a standard multicell structure, the accelerating mode is the $\pi$ - 0 mode ( $\pi$ cell-to-cell phase advance and 0 subunit-tosubunit phase advance). The second difference from a standard long multicell structure is the very weak coupling of subunits, $k_{s s}$. It is smaller roughly by a factor of 50 than cell-to-cell coupling. The energy flow via this coupling was the main objective in the beam test. Although the $k_{s s}$ coupling is very weak we can control the stored energy (gradient) in each subunit by means of cold tuners, which have high frequency resolution (nowadays better than $1 \mathrm{~Hz}$ ). A further difference is as follows. The $\pi-0$ mode is below cutoff in interconnecting tubes. This makes possible the attachment of higher order mode (HOM) coupler(s) at interconnection(s), in the "middle" 


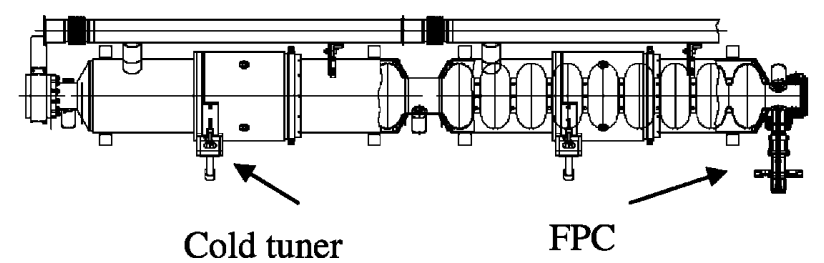

FIG. 1. SST-II: the left subunit is assembled in a He vessel with cold tuner. The right one is shown with the single FPC needed to transfer rf power to 18 cells.

of the multicell structure. In this way, good damping of parasitic modes can be maintained, avoiding strong heating of the couplers. The first superstructure (SST-I), as proposed in [1], was meant to be made of four 7-cell cavities. We have built a $\mathrm{Cu}$ model of this version and six $\mathrm{Nb} 7$-cell subunits, of which the best performing four would be assembled in the superstructure. The assembly would be done by means of superconducting gaskets. Meanwhile, a $2 \times 9$-cell version (SST-II, see Fig. 1) has been studied and found to be more attractive for the TESLA collider. This version keeps the same fill factor of the tunnel as the first one. SST-II is shorter and its production, cleaning, and handling will be easier. Savings in the investment cost are of the same order for both superstructures. The first version reduces the number of FPCs to $33 \%$ of the number needed for the base design. Since subunits are shorter (seven cells, not nine cells), it demands more liquid He vessels and cold tuners. The second version, SST-II, reduces the number of FPCs to $50 \%$ but it keeps the same number of He vessels and tuners as the base layout (if no additional structures are installed in the linac to fill up saved space). Table I compares three

TABLE I. Number of structures and their auxiliaries for three layouts of the $500 \mathrm{GeV}$ TESLA collider (without $2 \%$ energy overhead).

\begin{tabular}{lccc}
\hline \hline Layout & FPCs & $\begin{array}{c}\text { HOM } \\
\text { couplers }\end{array}$ & $\begin{array}{c}\text { Cold tuners, } \\
\text { He vessels }\end{array}$ \\
\hline 9-cells & 20592 & 41184 & 20592 \\
SST-I & 7032 & 35160 & 28128 \\
SST-II & 10926 & 43704 & 21852 \\
\hline \hline
\end{tabular}

TABLE II. The rf parameters of both superstructures.

\begin{tabular}{lcc}
\hline \hline Parameter & SST-I & SST-II \\
\hline Number of cells in subunit & 7 & 9 \\
Number of subunits & 4 & 2 \\
$(R / Q)$ per subunit $(\Omega)$ & 732 & 985 \\
$E_{\text {peak }} / E_{\text {acc }}$ & 2 & 2 \\
$B_{\text {peak }} / E_{\text {acc }}[\mathrm{mT} /(\mathrm{MV} / \mathrm{m})]$ & 4.2 & 4.2 \\
$L_{\text {active }}(\mathrm{m})$ & 3.23 & 2.08 \\
\hline \hline
\end{tabular}

layouts for the other and the most favorable scenario, when the $500 \mathrm{GeV}$ stage length of the TESLA tunnel is fixed and the saved space is filled up with additional accelerating elements. The rf parameters of superstructures are listed in Table II.

\section{PREPARATION OF THE TEST}

\section{A. The $2 \times 7$-cell version}

For a few reasons we have "split" the $4 \times 7$-cell prototype into two $2 \times 7$-cell superstructures. The superconducting gaskets, needed to assemble the SST-I prototype, did not perform reliably when installed on 7-cell subunits. We could not reach stable accelerating gradients testing subunits in the vertical cryostat, even thought the results on a 2-cell test cavity were encouraging. Subunits of $2 \times 7$-cell prototypes could be welded together. Fortunately, the infrastructure for cavity preparation at DESY was able to accommodate $2.08 \mathrm{~m}$ long cavities without major changes. The second argument to split SST-I was similarity in the computed (HOMDYN, [3]) bunch-to-bunch energy variation of the $2 \times 7$-cell and $2 \times 9$-cell versions. For all bunches in the TESLA macropulse the computed variation is very small, $\pm 5 \times 10^{-5}$ for the $2 \times 9$-cell and $\pm 3 \times 10^{-5}$ for the $2 \times 7$-cell version (Fig. 2). The shape difference of two figures results only from the different modes beating.

The conclusion was that the beam test of already existing 7-cell subunits assembled in two $2 \times 7$-cell prototypes will tell us more about the favorable SST-II version, will benchmark our computation, and will give finally twice more statistics for the measured results.

\section{B. TTF linac}

Both $2 \times 7$-cell superstructures were assembled into a spare cryomodule and installed in the TESLA Test Facility (TTF) linac next to the injector. Many components limiting the aperture of the beam line which could

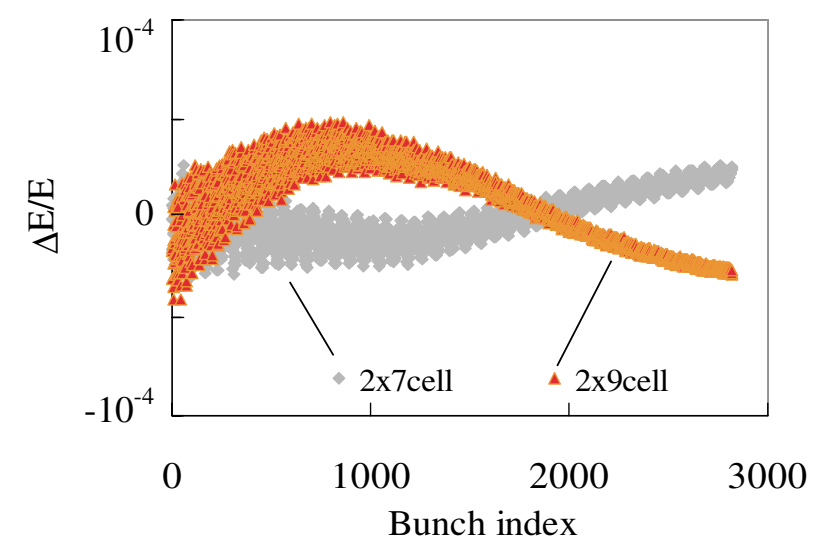

FIG. 2. (Color) Computed energy deviation for the TESLA beam: 2820 bunches, $3.2 \mathrm{nC} /$ bunch, spacing $337 \mathrm{~ns}$. 
influence our test were removed from the linac. Additional beam position monitors (BPMs) were installed behind and at the front of the dipole magnet next to the injector to measure the energy of the beam coming out of the injector. The energy measurement at the end of the linac was performed by means of the spectrometer dipole with two BPMs at its front and one BPM behind it. With this arrangement collective betatron motion could be extracted from the displacement measured behind the dipole. The highest estimated accuracy was $2 \times 10^{-4}$ and it was limited by the resolution of BPMs. It was better by a factor of 3 than for the operation of standard 9-cell structures during the entire time of experiments at the TTF linac in previous years. Because of a very intense experimental program at the TTF linac in the year 2002, a second cryomodule, housing eight 9-cell cavities, was installed simultaneously with the superstructures for a long-term performance test. The presence of this cryomodule had consequences for the test of the superstructures, as we will see later. Both cryomodules were cooled down in May 2002.

\section{THE TEST}

The planned cold and beam test was expected to answer the following three questions:

How to balance the gradient in subunits?

How stable is the energy gain?

How good is damping of the HOMs?

We will discuss the answers to these questions in the next three sections.

\section{A. Balance of the stored energy in subunits}

The field profiles of the accelerating mode of both superstructures had been measured with the help of the bead-pull (perturbation) technique before the final chemical treatment and the final high pressure water rinsing were done. Amplitudes of the accelerating field on axis of both prototypes (P1, P2) are shown in Fig. 3. Both had good field flatness, better than $92 \%$ and $94 \%$, respectively. At that stage, the subunits were already equipped with He vessels and there was no access to the cells to make further improvement in the field profile. One may expect that any additional chemical treatment or even cooldown could change the field profile. It was worthwhile to estimate this change experimentally. As usual, after the final chemical polishing and water rinsing there is no more possibility to use the bead-pull technique to measure and to adjust the fields. It is always a risk to contaminate a cleaned superconducting surface by moving a perturbing bead and its holding string in the interior of a cavity. Still, one can apply the perturbation method to balance the mean gradient in both subunits using the cold tuners instead of a bead to perturb the electromagnetic (em) fields. Both prototype's subunits have the same stored energy (or mean gradient) when the same pertur- (a)

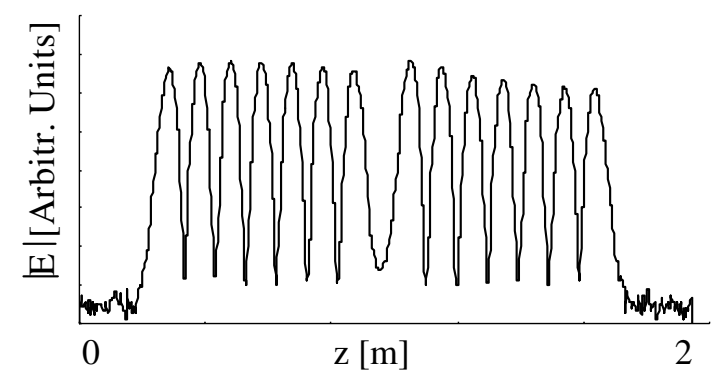

(b)

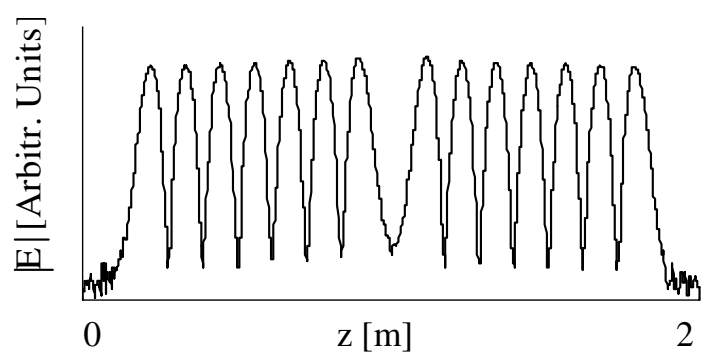

FIG. 3. Peak-to-peak field flatness. (a) P1, 92\% and (b) P2, 94\%.

bation causes the same frequency shift. For this, the cold tuner of each subunit was moved by 1000, 2000, and 5000 steps and for each position the frequency change $\Delta f$ of the $\pi-0$ mode was measured. Then, the final positions of the tuners were chosen to maintain exact frequency equal to $1.3 \mathrm{GHz}$ of the $\pi$-0 mode and simultaneously to ensure that the subunits show the same $\Delta f$ when their tuners are moved by the same number of steps. The final status of the prototypes was cross-checked in the following way. We compared, for each cold prototype, the fundamental passband frequencies with the frequencies measured at room temperature when the bead-pull method showed the best achievable field profile. The deviation from an ideal linear shift of frequencies is very sensitive to relative detuning of subunits and/or frequency perturbation of individual cells (due to possible nonuniform chemistry or nonuniform shrinkage). It is a very good indicator of any change in the field profile. The conclusion drawn from the test of this method and its application to the standard 9-cell cavities was that when the relative deviation of frequencies was below $1 \times 10^{-5}$, the change in the field flatness was less than $2 \%$. The deviation we measured for both prototypes (Fig. 4) was below $8 \times 10^{-6}$, so we concluded that field profiles remained unchanged after the final chemistry, high pressure water rinsing, and assembly in the cryostat, and after the cooldown procedure.

\section{B. Energy gain stability}

This experiment was the "proof of principle" test. As was mentioned already, our main concern was the energy flow via very weak coupling between subunits. The stability of the energy gain for all bunches in the train means 


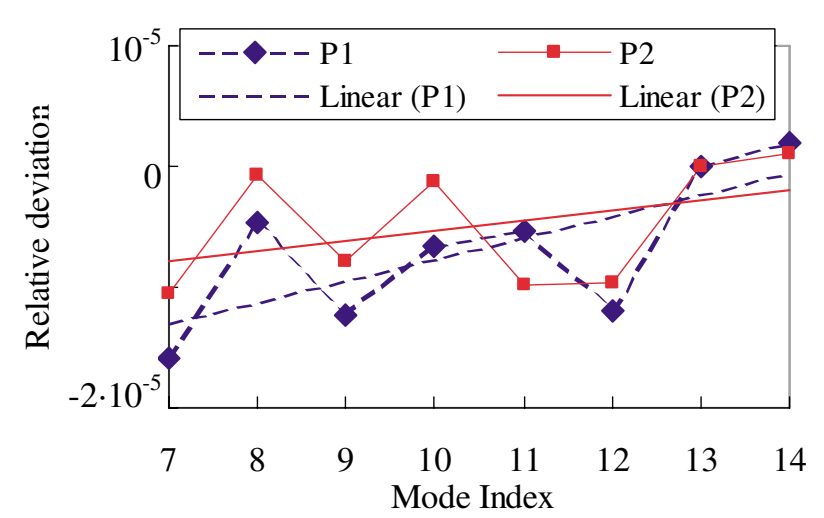

FIG. 4. (Color) Relative deviations (zigzag lines) from ideal linear spectrum shifts (straight lines) as measured for the eight highest modes of both prototypes.

that cells' stored energy is refilled in time between two consecutive bunches. The test was performed in two parts. In the first one, we examined the prototypes to a slow decay of the stored energy during the acceleration. In the second part we measured directly bunch-to-bunch energy modulation at the end of the linac. In both parts of this test neither prototypes nor injector were pushed to their limits in performance to keep the operation of the linac very stable. Both prototypes could be operated very reliably at $15 \mathrm{MV} / \mathrm{m}$ (the subunits had not been thermally treated at $1400^{\circ} \mathrm{C}$ ). The TTF injector was designed in 1997, when the TESLA beam parameters were different from those optimized later and proposed in the Technical Design Report (TDR) in 2001. The operation of the injector, with the smallest charge fluctuation of $2.8 \%$ within the macropulse, was possible when bunch charge did not exceed $4 \mathrm{nC}$. The bunch spacing of $t_{b}=1 \mu \mathrm{s}$ (the same as for the very first TESLA beam) had been chosen to meet the highest sampling rate of the implemented BPMs's electronics.

The HOMDYN prediction for this TTF beam was that bunch-to-bunch energy variation is in the same range as shown in Fig. 2. The reflection-free operation, when the beam current is $4 \mathrm{~mA}$ and the gradient is $15 \mathrm{MV} / \mathrm{m}$, demanded the $Q_{\text {load }}$ of the FPCs to be $4.2 \times 10^{6}$. The rise time of em fields resulting from this $Q_{\text {load }}$ value was $790 \mu \mathrm{s}$ and the longest beam on time was limited to $530 \mu \mathrm{s}$ (klystron pulse length was in total $1.32 \mathrm{~ms}$ ).

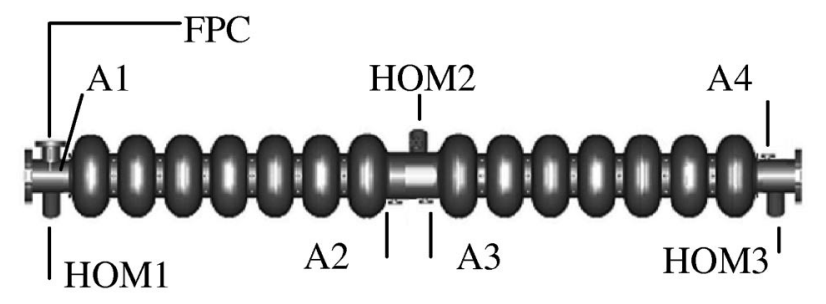

FIG. 5. Prototype of $2 \times 7$ cells. Field probes: A1-A4. HOM couplers: HOM1-HOM3.

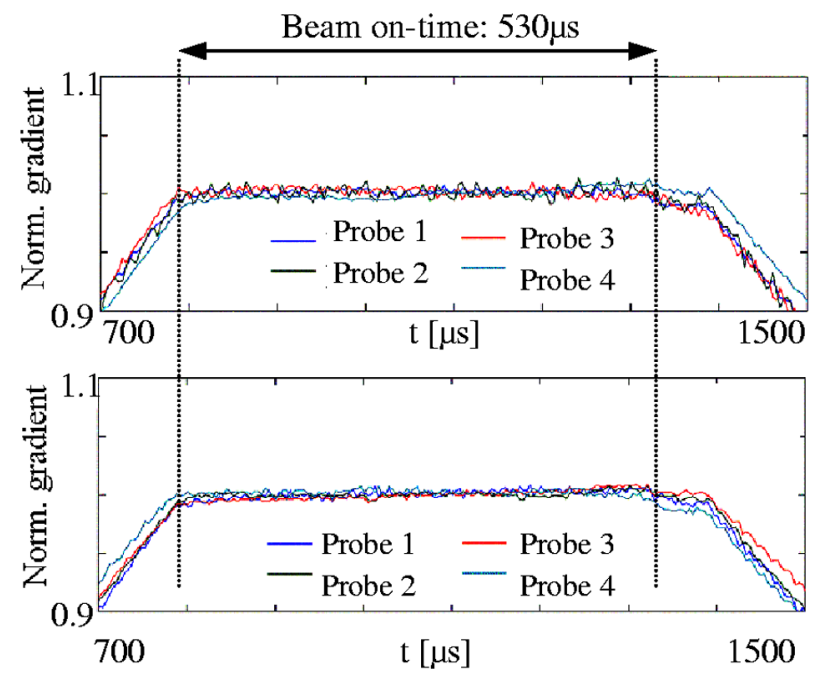

FIG. 6. (Color) Signals from field probes (P1 upper diagram, P2 lower diagram) measured during the acceleration of 530 bunches, $q=4 \mathrm{nC}, t_{b}=1 \mu \mathrm{s}$ at $15 \mathrm{MV} / \mathrm{m}$.

Each prototype was equipped with four field probes, placed one near each end cell (see Fig. 5). They were used to monitor the field strength during the acceleration.

An example of measured signals is presented in Fig. 6. Without the energy refilling the beam would take almost $70 \%$ of the energy stored in cells and the voltage would drop by $45 \%$. No such phenomenon was observed. Both diagrams confirm the expectation that energy flow via weak coupling is sufficient. Even the signals of probes No. 3 and No. 4 (located at the subunit without FPC) in both prototypes did not decay during the acceleration. All signals had some noisy fluctuations. The strongest oscillation was at $250 \mathrm{kHz}$. It was caused by down-converters of the low level rf system controlling the phase and the amplitude of accelerating fields. Zoomed signals of the P2

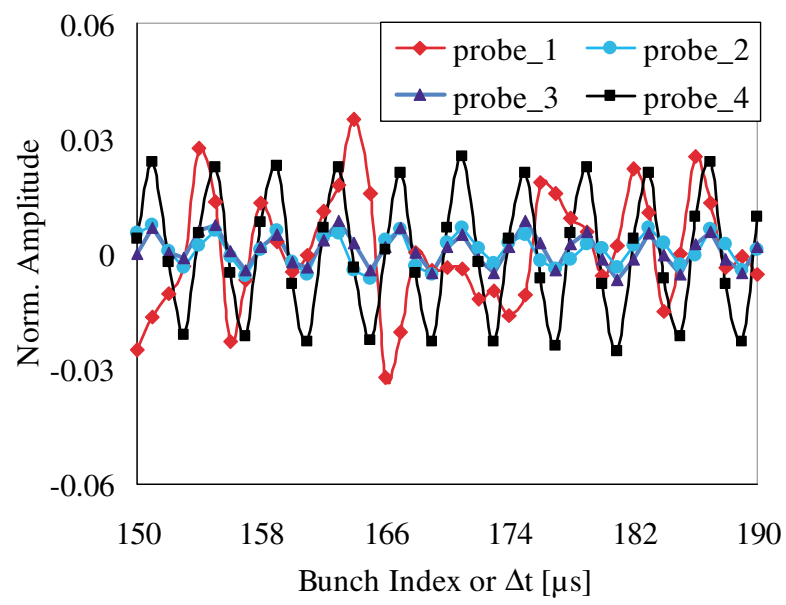

FIG. 7. (Color) Prototype P2. Oscillations of signals (normalized) during the acceleration. 


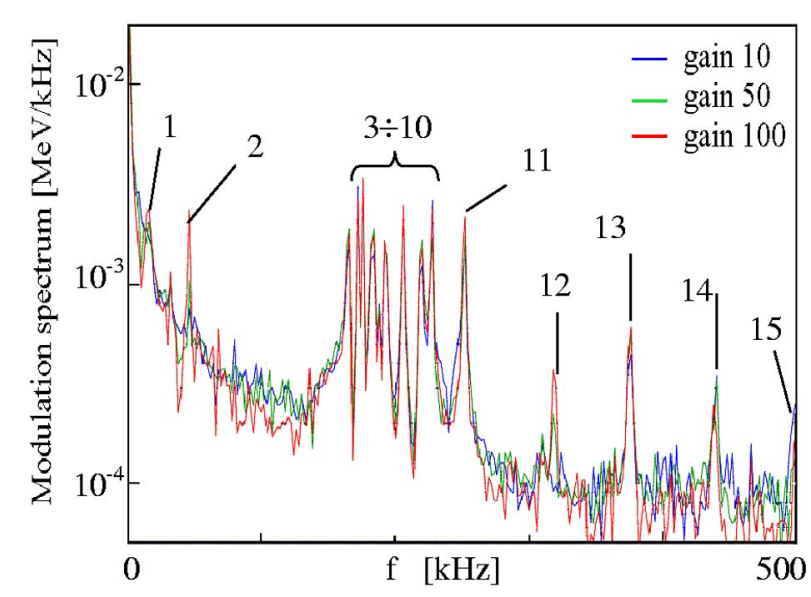

FIG. 8. (Color) Spectrum of the energy modulation as measured at the end of the linac.

prototype, in the time range $150-190 \mu$ s after the beam was switched on, are shown in Fig. 7. All signals in this figure are normalized: $(A-\langle A\rangle) /\langle A\rangle$. They showed the oscillation at $250 \mathrm{kHz}$ (4 $\mu$ s period) which is superimposed on the other oscillations, well seen in the signal of probe No. 1. The $250 \mathrm{kHz}$ modulation was also seen during operation of the standard 9-cell cavities. We found, in the second part of the experiment, six more oscillations caused by the feedback loops.

As was mentioned, the second part of this experiment was devoted to measuring directly at the end of the linac the bunch-to-bunch energy variation. The Fourier transformation of three signals (from the BPM behind the dipole), measured for three different gains in the feedback loop, is shown in Fig. 8. One can see 15 oscillations in total, which reacted to the gain change in very different ways. Peaks No. 1, No. 2, No. 12, and No. 13 increased when the loop gain increased. Peaks No. 14 and No. 15 decreased versus the gain. All other peaks remained unchanged, including the $250 \mathrm{kHz}$ one, which we discussed above. Seven peaks were due to the feedback loops; eight (Nos. 3-10) were caused by the second cryomodule. All eight cavities of this cryomodule were detuned from the $1.3 \mathrm{GHz}$ frequency by roughly $200 \mathrm{kHz}$ and no power was delivered to these cavities during the entire energy gain test. Still, the beam induced voltage in these cavities modulated the energy of bunches. We could change the frequency of an individual peak by driving the cold tuner of the corresponding cavity. Finally, the conclusion from the energy stability test was that no slow gradient decay and no modulation caused by superstructure prototypes was seen within the accuracy limit in the measurement of $2 \times 10^{-4}$ [4]. One should mention here that this result proves that superstructures fulfill the TDR specification for bunch-tobunch energy variation, which must be below $5 \times 10^{-4}$.

\section{HOM damping}

Each prototype had three HOM couplers (Fig. 5) which had been attached to the end beam tubes and to the interconnection. The SST-II version will have four more cells and we plan to attach two HOM couplers at the interconnection to compensate for that. We will report on the results we measured for the transverse modes, since these modes are relevant for the quality of the TESLA beam. Three methods were applied to measure the frequency and impedance, $Z=(R / Q) Q_{\text {ext }}$, of HOMs. At first, we measured the modes' frequency and $Q_{\text {ext }}$ by means of network analyzers. For both prototypes, we measured modes up to $3.2 \mathrm{GHz}$ including the five dipole passbands and the lowest passbands of monopole, quadrupole, and sextupole modes. In total, 420 modes have been measured. The method gives the impedance when one assumes that actual $(R / Q)$ equals its computed value. The method is limited to "well-isolated" modes. The error in frequency measurement increases when $Q_{\text {ext }}$ of a mode gets lower and when neighboring modes overlap. The frequency errors were misleading for the search of some modes we wanted to check with two other methods.

The second method we applied was the active mode excitation [5]. Modes with potentially high impedance were excited via one of the HOM couplers by means of a $50 \mathrm{~W}$ amplifier. By controlling the power coupled out by two other HOM couplers we estimated deflection of the on axis injected beam. It was compared to the value measured in the BPM, $15 \mathrm{~m}$ downstream from the cryomodule. The method potentially can give all parameters of an excited mode, $Z$ and the polarization, if deflection is measured in both $x$ and $y$ directions. It is sensitive to setting of the beam line optics between the cryomodule and the BPM. One can apply this method to modes which couple well to HOM couplers. Forty-seven modes were measured with the active method. Examples of measured BPM signals are shown in Figs. 9 and 10.
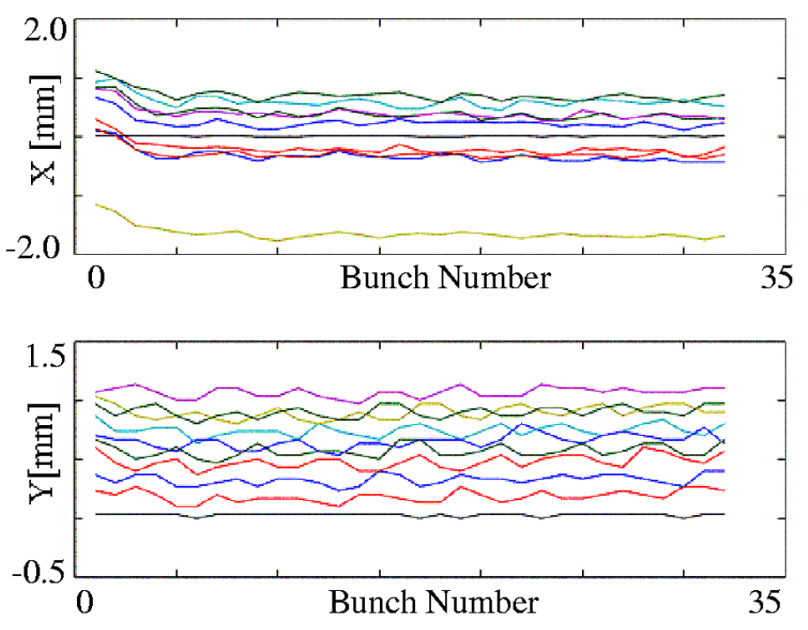

FIG. 9. (Color) BPM signals without the excitation of the deflecting dipole mode. 

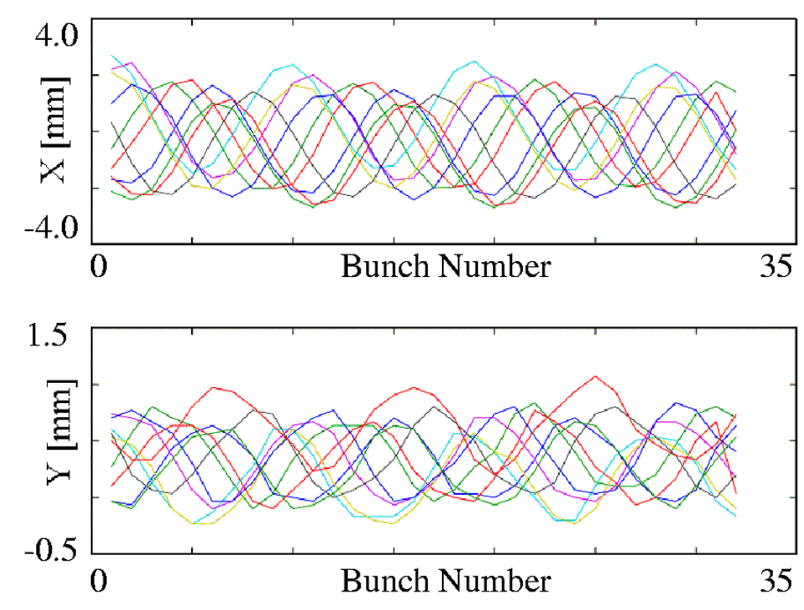

FIG. 10. (Color) BPM signals with the excitation of the deflecting dipole mode. Note different scales for both signals in the $x$ plane.

In this particular case one polarization of the highest impedance dipole $\left(R / Q=27 \Omega / \mathrm{cm}^{2}\right)$ at $f=$ $2573.971 \mathrm{MHz}$ was excited with $20 \mathrm{~W}$ forward power. The damping of this mode was very good. Its $Q_{\text {ext }}$ was only $2.1 \times 10^{4}$ (5 times below the specification). We measured the deflection in both directions for 32 bunches $(2 \mathrm{nC})$ in a $32 \mu \mathrm{s}$ long pulse. Ten consecutive pulses are shown in each figure. The signals measured without the excitation (Fig. 9) indicated that bunch position in both planes was varying about $\pm 0.6 \mathrm{~mm}$ from pulse to pulse (one exception was observed for the $x$ direction), although, within one pulse, the position stability without the excitation was 1 order of magnitude better. Strong oscillation of the beam position was observed when the amplifier was on (Fig. 10). The mode has been tested 6 times, for various settings of the optics elements and for various HOM couplers used to transfer rf power into the cavity. The measured and the computed deflections are displayed in Fig. 11. The differences were mainly due to the optics whose optimum setting should minimize its influence on the trajectory. The estimation of the beam deflection $\left(R_{-}\right.$comp) was done with the assumption that the beam drifts between the cryomodule and the BPM. The second reason for the discrepancy, relevant for modes propagating in beam lines, was the direct coupling of a part of the rf power into the beam line [6]. This made the estimation of the deflecting fields less accurate since an unknown part of the power was radiated directly from the coupler. The mode we discuss in the example is above cutoff and the best method was to use the HOM coupler attached to the interconnection between two subunits to avoid direct radiation into the beam lines. The mean value of the measured deflections was $\langle R\rangle=1.8 \mathrm{~mm}$ and its computed value was $R_{-}$comp $=1.7 \mathrm{~mm}$. The measured polarization is shown in Fig. 12. The mean value, which was found for the cold prototype, was $73^{\circ} \pm 10^{\circ}$ (angle measured $\mathrm{cw}$ from the $y$ direction). The differences were

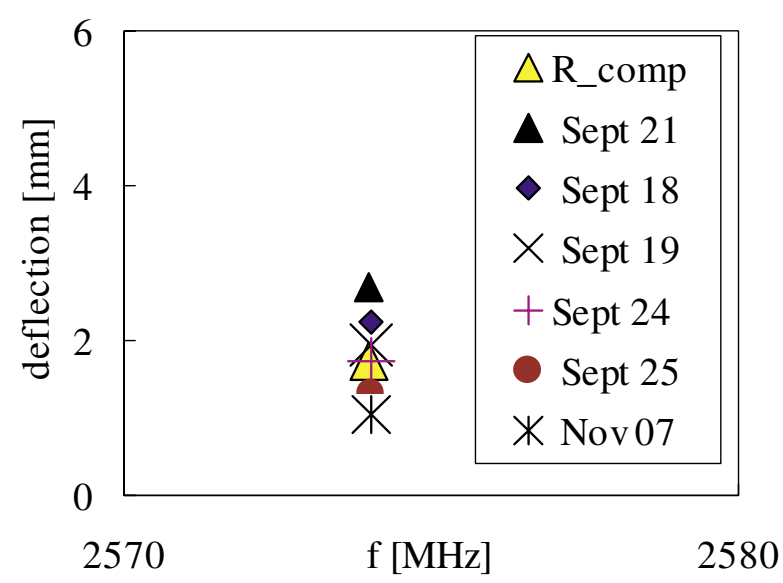

FIG. 11. (Color) Deflection measured for the different optics setting.

mainly due to calibration errors of the BPM signals in both planes. We could not reliably measure angular position of this mode when the prototype was warm since both polarizations overlapped very strongly. Nevertheless, the measured deflection gave the estimation of $Z$, which in the worst case is 2 times higher than expected from the network analyzer measurements and which still would be harmless to the TESLA beam. The polarization measurement showed that this mode, when excited by the accelerated beam, will deflect it almost horizontally. We measured other modes in a similar way.

The third method we applied to measure $Z$ was based on the HOM excitation by the accelerated beam when it passes the cavity off axis. The charge of the beam was modulated to hit modes with sideband spectral lines generated in this way. The method was previously applied twice to the standard cavities. Here we will not discuss its results, which are presented in more detail in [7]. All

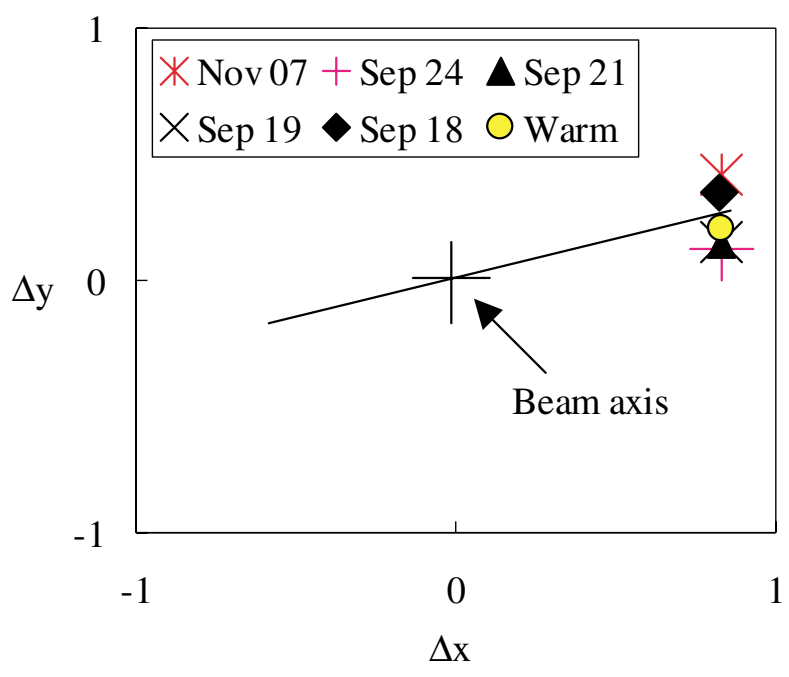

FIG. 12. (Color) Normalized position of the deflected beam in the $x-y$ plane. 
three methods proved good damping of HOMs. Damping of dipole modes with $(R / Q)>1 \Omega / \mathrm{cm}^{2}$ is shown in Fig. 13. All modes relevant for the TESLA collider, up to $2.58 \mathrm{GHz}$, are damped by a factor 5 to 100 better than the specification $\left(Q_{\mathrm{ext}} \leq 10^{5}\right)$. The damping of dipoles with $(R / Q)<1 \Omega / \mathrm{cm}^{2}$ and other transverse modes is also good (Fig. 14). We have found a few modes only (in the 5th passband, $\sim 3.08 \mathrm{GHz}$ ), among 420 measured modes, with $Q_{\text {ext }}=10^{7}-2 \times 10^{8}$. Their $(R / Q)$ s are almost zero and thus they cannot degrade the quality of the TESLA beam. The groups of quadrupoles $(\sim 2.3 \mathrm{GHz})$ and sextupoles $(\sim 2.7$ and $\sim 3.06 \mathrm{GHz})$ with $Q_{\text {ext }} \sim 10^{6}$ are also irrelevant for the TESLA beam dynamics since their impedances scale versus trajectory offset with 4th $(n=2)$ and 6th $(n=3)$ powers, respectively.

\section{FINAL REMARKS}

\section{A. Summary of the experiment}

The cold and beam test of both prototypes has confirmed that one can use weakly coupled structures for the acceleration. Neither beam energy modulation, slow gradient decay, nor insufficient HOMs damping, resulting from the coupling of two subunits, have been observed. The stability of the bunch-to-bunch energy gain was measured within the limit of the beam diagnostics in the TTF linac. Although, the accuracy of the energy gain measurement has not reached the level of the theoretical estimation, which was an order of magnitude smaller, the experiment showed that the TESLA specification already has been fulfilled. We have demonstrated two methods to balance the gradient in the weakly coupled subunits. The agreement of both methods was good and both confirmed that final chemical cleaning may be performed without additional degradation in the field flatness.

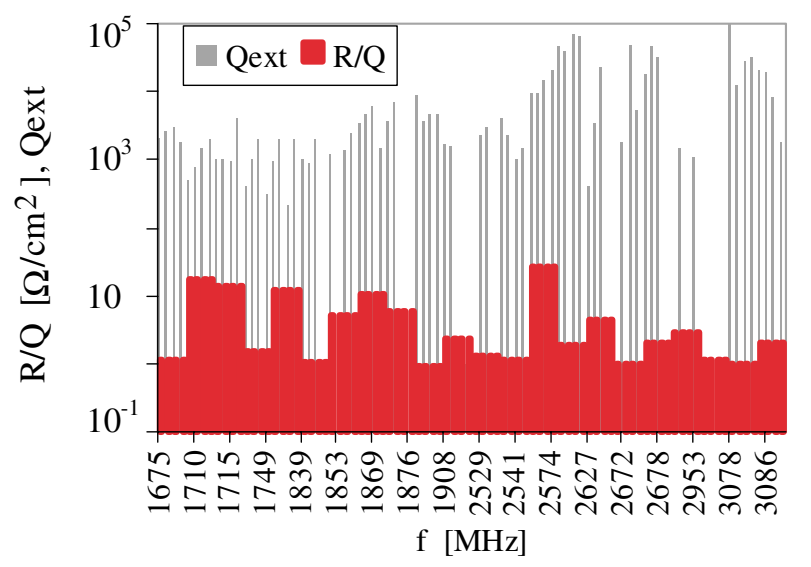

FIG. 13. (Color) Damping of dipoles with $(R / Q) \geq 1 \Omega / \mathrm{cm}^{2}$.

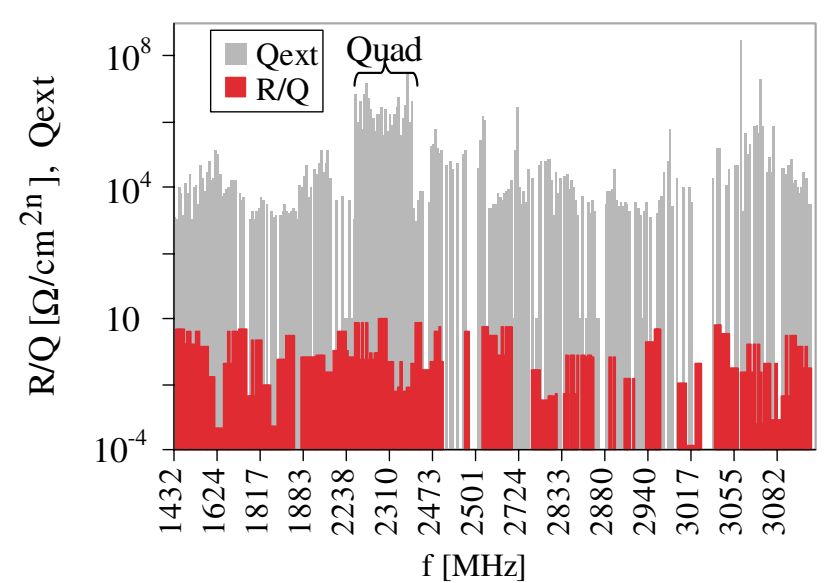

FIG. 14. (Color) Damping of modes with $(R / Q)<1 \Omega / \mathrm{cm}^{2 n}$.

The experiment showed that the electronics for phase and amplitude control, used routinely to operate standard 9-cell cavities in the TTF linac, can be applied to operate superstructures. Further improvement of the control system seems to be possible to provide better suppression of the modulations coming from the control system itself.

\section{B. Production and future}

The production and preparation of superstructures is more challenging than for standard cavities, but obviously not impossible, especially if the infrastructure for welding, pretuning, and cleaning is planned from the very beginning to accommodate longer structures.

Now the next logical step would be to demonstrate the performance of the $2 \times 9$-cell superstructure. After the beam experiment on $2 \times 7$ cells we are convinced that this can be only a challenge for the production, but no fundamental physical problems due to very weak coupling are expected. We will continue measurements on $\mathrm{Cu}$ models of SST-II to optimize the angular position of HOM couplers. At the moment the beam experiment on a $\mathrm{Nb}$ prototype is neither scheduled nor funded. Meanwhile, a new application of the superstructure concept has been proposed and at present is under study [8]. The very good HOM damping makes superstructures with fewer cells an attractive alternative to standard accelerating structures for a high current linac driving free-electron lasers. The next application to energy recovery linacs will be studied in more detail in the future.

\section{ACKNOWLEDGMENTS}

We would like to express our gratitude to the TESLA collaboration group for many helpful discussions and to all operators of the TTF linac for their help during the experiment. 
[1] J. Sekutowicz, M. Ferrario, and C. Tang, in Proceedings of the LC'97, Zvenigorod, 1997.

[2] J. Sekutowicz, M. Ferrario, and C. Tang, Phys. Rev. ST Accel. Beams 2, 062001 (1999).

[3] M. Ferrario, A. Mosnier, L. Serafini, F. Tazzioli, and M. Tessier, Part. Accel. 52, 1 (1996).

[4] H. Schlarb, V. Ayvazyan, P. Castro, M. Ferrario, M. Hüning, R. Kammering, S. Schreiber, J. Sekutowicz, S. Simrock, and M. Wendt, in Proceedings of the PAC'03, Portland, 2003 (to be published).
[5] J. Sekutowicz, A. Gössel, G. Kreps, and S. Zheng, in Proceedings of the PAC'03, Portland, 2003 (Ref. [4]).

[6] M. Dohlus (private communication).

[7] P. Castro, N. Baboi, G. Devanez, A. Gössel, S. Schreiber, J. Sekutowicz, and M. Wendt, in Proceedings of the PAC'03, Portland, 2003 (Ref. [4]).

[8] J. Sekutowicz, P. Kneisel, C. Thomas, G. Wu, and S. Zheng, in Proceedings of the PAC'03, Portland, 2003 (Ref. [4]). 